\title{
Microfluidic microcalorimetry of Acid/Base reaction using FTIR Thermospectroscopy imaging
}

\author{
by S. Chevalier ${ }^{\star}$, J.-N. Tourvieille**, A. Sommier*, J.-C. Batsale* and C. Pradere ${ }^{*, * *}$
}

* I2M Institute, UMR CNRS 5295, esplanade des arts et métiers, 33405 Talence cedex, France, stephane.chevalier@u-bordeaux.fr, alain.sommier@u-bordeaux.fr, jean-christophe.batsale@u-bordeaux.fr and christophe.pradere@u-bordeaux.fr

** Solvay/Laboratoire du Futur , 178, avenue du Docteur Schweitzer, 33608 Pessac, France, Tourvieille, jeannoel.tourvieille@solvay.com

\begin{abstract}
Quantitative understanding of heat and mass transfers during chemical reactions occurring between different fluids is of prime interest for fundamental chemical physics and applied microtechnologies such as lab-on-a-chip synthesis and fuel cells [1]. To analyse the diffusion and reaction processes, microfluidic chips appear to be powerful tools as the mass transport and the kinetics of reactions can be controlled. These technologies are also well suited to perform imaging studies such as thermal and concentration field measurements in reactive processes using an IR camera associated to spectroscopic measurements with monochromatic IR sources [2,3]. Use in combination with inverse methods based on reaction diffusion equations, imaging methods in microfluidic chips will lead to the characterization of all heat and mass transfer properties in a reactive system. If some work was already done in the field of heat transfer [4], the objectives of the present study are to demonstrate that both heat and mass diffusivities, kinetics parameters, and in particular the standard enthalpy of reaction can be quantified in a hydrochloric acid and hydroxide sodium reaction in a microfluidic channel.
\end{abstract}

The standard enthalpy of reaction is defined as $\Delta H=\rho C_{p} T_{0} \tau_{R}^{T} /\left(c_{0} \tau_{R}^{C}\right)$ [J/mol] where $\rho$ is the density, $C_{p}$ is the thermal capacity, $T_{0}$ is the ambient temperature, and $c_{0}$ is the initial concentration of the reactants. These data are given by the experimental setup and the fluids used (in general water as solvent). Thus, it remains $\tau_{R}^{C}$ and $\tau_{R}^{T}$ which are the characteristic times of the kinetic and heat production during reaction, respectively. To measure them, a microfluidic imaging setup was built as depicted in Figure 1(a). It is made of an FTIR source which generates IR interferograms in each pixel of the camera. Once processed with a fast Fourier transform algorithm, these interferograms leads to the multispectral absorbance of products and reactants (see Figure 1(b)) from which the knowledge of the concentration distribution is obtained. On the mathematical side, the classical diffusion-reaction model of two reactants to one product in a microchannel [5] was used to mimic the chemical reaction as presented in Figure 1(c). Inverse methods were finally used to estimate $\tau_{T}$ and $\tau_{C}$.

First results were obtained using a hydrochloric acid and hydroxide sodium reaction which produces salted water (i.e. $\mathrm{HCl}$ $+\mathrm{NaOH} \rightarrow \mathrm{NaCl}+\mathrm{H}_{2} \mathrm{O}$ ). A small Y-shape microchannel (5 mm-wide by $30 \mathrm{~mm}$-long) was fabricated and imaged (absorbance and thermal fields). To ensure an accurate measurement of the characteristic time, the reaction-diffusion model was analysed in details, and it was found that $\tau_{R}^{C}$ can be only measured in the middle of the channel (see Figure $2(a)$ and (b)) in what it is called the reactive zone. In the diffusive zone, only the transport properties (diffusivity) can be measured using an analytical solution of the reaction diffusion equation in the limiting case of large Damkhöler. In this communication, the complete characterization of $\tau_{R}^{C}$ and $\tau_{R}^{T}$ will be presented as well as the heat and mass transport properties. Finally, the standard enthalpy of the reaction will be estimated illustrating the high performances of quantitative contactless measurements using IR imaging techniques.

\section{REFERENCES}

[1] J.W. Lee, M.-A. Goulet, E. Kjeang, Microfluidic redox battery, Lab Chip. 13 (2013) 2504.

[2] M. Romano, M. Ryu, J. Morikawa, J.C. Batsale, C. Pradere, Simultaneous microscopic measurements of thermal and spectroscopic fields of a phase change material, Infrared Phys. Technol. 76 (2016) 65-71.

[3] N. Kakuta, H. Yamashita, D. Kawashima, K. Kondo, H. Arimoto, Y. Yamada, Simultaneous imaging of temperature and concentration of ethanol-water mixtures in microchannel using near-infrared dual-wavelength absorption technique, Meas. Sci. Technol. 27 (2016) 115401.

[4] C. Pradere, M. Joanicot, J.-C. Batsale, J. Toutain, C. Gourdon, Processing of temperature field in chemical microreactors with infrared thermography, Quant. Infrared Thermogr. J. 3 (2006) 117-135.

[5] L. Gálfi, Z. Rácz, Properties of the reaction front in an $A+B=C$ type reaction-diffusion process, Phys. Rev. A. 38 (1988) 3151-3154. 

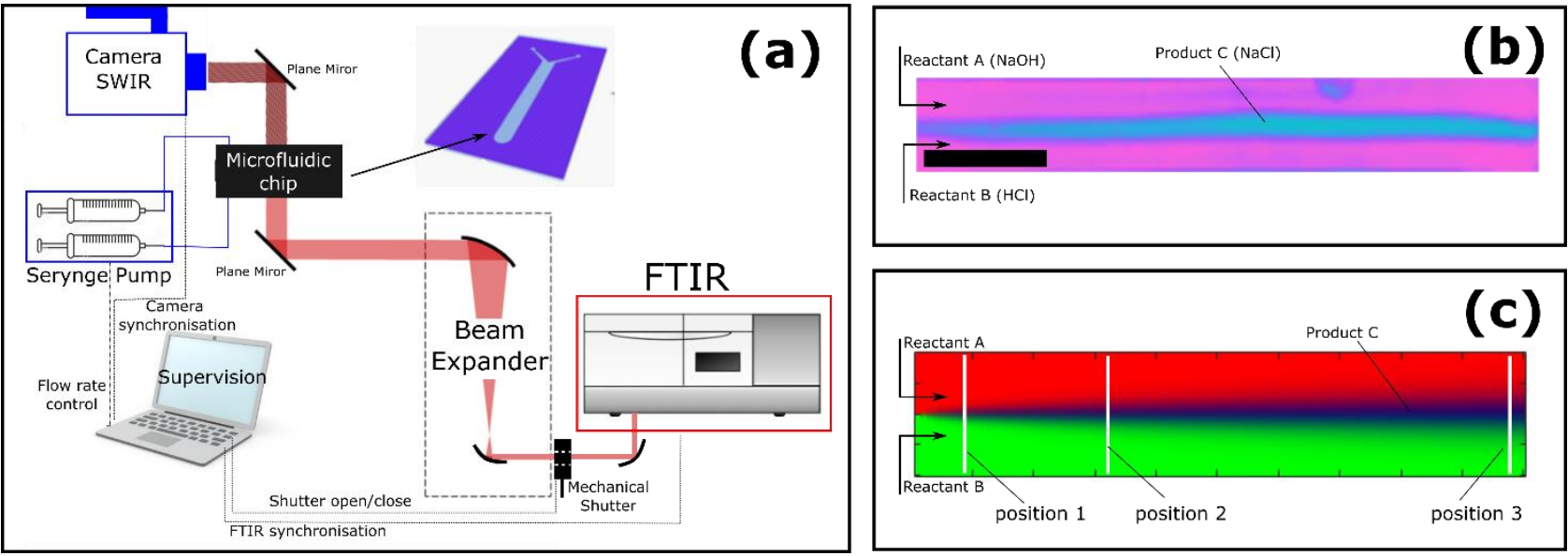

Fig. 1. (a) Experimental setup based on FTIR imaging to measure the multispectral absorbance and thermal field during the chemical reaction of two reactants. (b) Absorbance of the product ( $\mathrm{NaCl}$ ) in the microchannel. The scale bar is $5 \mathrm{~mm}$. (b) Numerical simulation of the reaction-diffusion of two reactants in a microchannel.

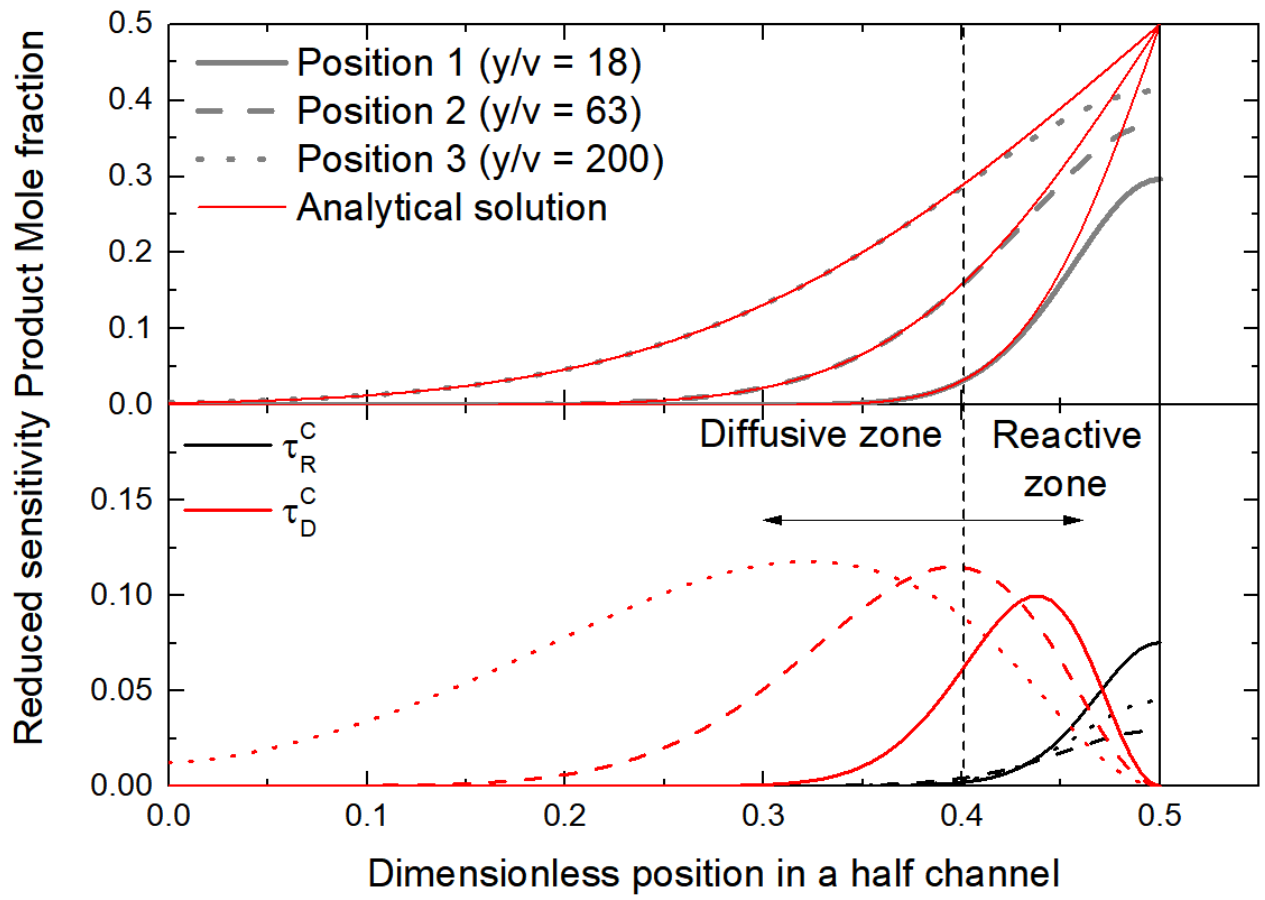

Fig. 2. Analysis of the reaction-diffusion model of product $C$ in a half channel. The vertical black solid line indicates the middle of the channel (a) Mole fraction distribution of the product $C$ for a range of positions in the channel (see Figure 1(c)). Comparison between the numerical and analytical solution. (b) Sensitivity analysis of $\tau_{R}^{C}$ and $\tau_{D}^{C}$ in the numerical model at the same channel positions. 